\title{
Learning Model-Based Sparsity via Projected Gradient Descent
}

\author{
Sohail Bahmani, Petros T. Boufounos, Member, IEEE, and Bhiksha Raj
}

\begin{abstract}
Several convex formulation methods have been proposed previously for statistical estimation with structured sparsity as the prior. These methods often require a carefully tuned regularization parameter, often a cumbersome or heuristic exercise. Furthermore, the estimate that these methods produce might not belong to the desired sparsity model, albeit accurately approximating the true parameter. Therefore, greedy-type algorithms could often be more desirable in estimating structuredsparse parameters. So far, these greedy methods have mostly focused on linear statistical models. In this paper we study the projected gradient descent with non-convex structured-sparse parameter model as the constraint set. Should the cost function have a Stable Model-Restricted Hessian the algorithm produces an approximation for the desired minimizer. As an example we elaborate on application of the main results to estimation in Generalized Linear Models.
\end{abstract}

\section{Index Terms}

Model-based sparsity, Estimation, Projected Gradient Descent, Stable Model-Restricted Hessian

\section{INTRODUCTION}

In a variety of applications such as bioinformatics, medical imaging, social networks, and astronomy there is a growing demand for computational methods that perform statistical inference on high-dimensional data. In the problems arising in these applications, $p$, the number of predictors in each sample is much larger than $n$, the number of observations. Although such problems are generally ill-posed, in many cases the data has known underlying structure such as sparsity that can be exploited to make the problem well-posed.

Beyond the ordinary, extensively studied, sparsity model, a variety of structured sparsity models have been proposed in the literature [1]-[8]. These sparsity models are designed to capture the interdependence of the locations of the non-zero components that is known a priori in certain applications. The models proposed for structured sparsity can be divided into two types. Models of the first type have a combinatorial construction and explicitly enforce the permitted "non-zero patterns" [4], [7]. Greedy algorithms have been proposed for the least squares regression with true parameters belonging to such combinatorial sparsity models [4]. Models of the second type capture sparsity patterns induced by the convex penalty functions tailored for specific estimation problems. Typically, such convex penalty functions are derived from convex relaxations of the combinatorial model. For example, consistency of linear regression with mixed $\ell_{1} / \ell_{2}$-norm regularization in estimation of group sparse signals having non-overlapping groups is studied in [1]. Furthermore, a different convex penalty to induce group sparsity with overlapping groups is proposed in [3]. In [5], using submodular functions and their Lovàsz extension, a more general framework for design of convex penalties that induce given sparsity patterns is proposed. In [8] a very general convex signal model is proposed that is generated by a set of base signals called "atoms". The model can describe not only plain and structured sparsity, but also low-rank matrices and several other low-dimensional models. We refer readers to [9], [10] for extensive reviews on the estimation of signals with structured sparsity.

In addition to linear regression problems under structured sparsity assumptions, nonlinear statistical models have been studied in the convex optimization framework [1], [2], [6], [11]. For example, using the signal model introduced in [8], minimization of a convex function obeying a restricted smoothness property is studied in [11] where a coordinate-descent type of algorithm is shown to converge to the minimizer at a sublinear rate. In this formulation and other similar methods that rely on convex relaxation one needs to choose a regularization parameter to guarantee the desired statistical accuracy. However, choosing the appropriate value of this parameter may be intractable. Furthermore, the convex signal models usually provide an approximation of the ideal structures the estimates should have, while in certain tasks such as variable selection solutions are required to exhibit the exact structure considered. Therefore, in such tasks, convex optimization techniques may yield estimates that do not satisfy the desired structural properties, albeit accurately approximating the true parameter. These shortcomings motivate application of combinatorial sparsity structures in nonlinear statistical models, extending prior results such as [4] that have focused exclusively on linear models.

Among the non-convex greedy algorithms, a generalization of Compressed Sensing is considered in [12] where the measurement operator is a nonlinear map and the union of subspaces is assumed as the signal model. This formulation, however, admits only a limited class of objective functions that are described using a norm. Furthermore, [13] proposes a generalization of the Orthogonal Matching Pursuit algorithm [14] that is specifically designed for estimation of group sparse

S.B. is with the School of Electrical and Computer Engineering at Georgia Institute of Technology.

P.B. is with Mitsubishi Electric Research Labs.

B.R. is with the Language Technologies Institute and the Department of Electrical and Computer Engineering at Carnegie Mellon University. 
parameters in Generalized Linear Models (GLMs). Also, [15] studies the problem of minimizing a generic objective function subject to plain sparsity constraint from the optimization perspective. Based on certain necessary optimality conditions for the sparse minimizer, some characterizations of sparse stationary points of the optimization problem are proposed in [15]. Then a few iterative algorithms are shown to converge to these stationary points, should the objective satisfies certain conditions. In parallel to our work, [16] has examined a variation of this problem, and provided similar results and guarantees. Specifically, in [16] the domain of the objective function is allowed to be an infinite-dimensional Hilbert space, whereas we only assume finitedimensional Hilbert spaces. The sufficient conditions introduced in [16] is essentially equivalent to our sufficient conditions, and both characterize the conditioning of second-order derivatives of the objective when restricted to subspaces of interest. The mentioned condition number controls the contraction factor in iterations of the algorithm in both [16] and our work. However, to establish the convergence, [16] requires the condition number to be smaller than $4 / 3$, which is more stringent than our results that, depending on what is known about the restricted second derivative, only require the condition number to be smaller than 3 or $3 / 2$. Furthermore, the accuracy of the method in [16] is only measured with respect to the model-consistent minimizer and the corresponding approximation error is expressed in terms of the value of the objective at certain minimizers (with different model parameters).

In this paper we study the projected gradient descent method, also a greedy algorithm, to approximate the minimizer of a cost function subject to a model-based sparsity constraint. Our approach can be applied to a broad set of problems, where the objective functions are not limited to quadratic functions or other norm-based functions assumed in most of the previous studies. The algorithm is described in Section [I] The sparsity model considered in this paper is similar to the models in [4] with minor differences in the definitions. To guarantee the accuracy of the algorithm our analysis requires the cost function to have a Stable Model-Restricted Hessian (SMRH) as defined in Section III. Using this property we can bound the distance of each iterate to any given reference point in the considered model by the sum of two terms. The first term, shrinks geometrically by the iterations, whereas the second term is a fixed approximation error that depends on the choice of the reference point. As an example, Section $[1 \mathrm{I}$ considers the cost functions that arise in Generalized Linear Models and discusses how the proposed sufficient condition (i.e., SMRH) can be verified and how large the approximation error of the algorithm is. To make precise statements on the SMRH and on the size of the approximation error we assume some extra properties on the cost function and/or the data distribution. Finally, we discuss and conclude in Section $\mathrm{V}$.

Notation.: In the remainder of the paper we denote the positive part of a real number $x$ by $(x)_{+}$. For a positive integer $k$, the set $\{1,2, \ldots, k\}$ is denoted by $[k]$. Vectors and matrices are denoted by boldface characters and sets by calligraphic letters. The support set (i.e., the set of non-zero coordinates) of a vector $\mathbf{x}$ is denoted by $\operatorname{supp}(\mathbf{x})$. Restriction of a $p$-dimensional vector $\boldsymbol{v}$ to its entries corresponding to an index set $\mathcal{I} \subseteq[p]$ is denoted by $\left.\mathbf{v}\right|_{\mathcal{I}}$. Similarly $\mathbf{A}_{\mathcal{I}}$ denotes the restriction of a matrix $\mathbf{A}$ to the rows enumerated by $\mathcal{I}$. For square matrices $\mathbf{A}$ and $\mathbf{B}$ we write $\mathbf{B} \preccurlyeq \mathbf{A}$ to state that $\mathbf{A}-\mathbf{B}$ is positive semidefinite. We denote the power set of a set $\mathcal{A}$ as $2^{\mathcal{A}}$. For two non-empty families of sets $\mathcal{F}_{1}$ and $\mathcal{F}_{2}$ we write $\mathcal{F}_{1} \cup \mathcal{F}_{2}$ to denote another family of sets given by $\left\{\mathcal{X}_{1} \cup \mathcal{X}_{2} \mid \mathcal{X}_{1} \in \mathcal{F}_{1}\right.$ and $\left.\mathcal{X}_{2} \in \mathcal{F}_{2}\right\}$. Moreover, for any non-empty family of sets $\mathcal{F}$ for conciseness we set $\mathcal{F}^{j}=\mathcal{F} \cup \ldots \cup \mathcal{F}$ where the operation $\cup$ is performed $j-1$ times. The inner product associated with a Hilbert space $\mathcal{H}$ is written as $\langle\cdot, \cdot\rangle$. The norm induced by this inner product is denoted by $\|\cdot\|$. We use $\nabla f(\cdot)$ and $\nabla^{2} f(\cdot)$ to denote the gradient and the Hessian of a twice continuously differentiable function $f: \mathcal{H} \mapsto \mathbb{R}$. For an index set $\mathcal{I} \subset[p]$ with $p=\operatorname{dim}(\mathcal{H})$, the restriction of the gradient to the entries selected by $\mathcal{I}$ and the restriction of the Hessian to the entries selected by $\mathcal{I} \times \mathcal{I}$ are denoted by $\nabla_{\mathcal{I}} f(\cdot)$ and $\nabla_{\mathcal{I}}^{2} f(\cdot)$, respectively. Finally, numerical superscripts within parentheses denote the iteration index.

\section{Problem Statement and Algorithm}

To formulate the problem of minimizing a cost function subject to structured sparsity constraints, first we provide a definition of the sparsity model. This definition is an alternative way of describing the Combinatorial Sparse Models in [7]. In comparison, our definition merely emphasizes the role of a family of index sets as a generator of the sparsity model.

Definition 1. Suppose that $p$ and $k$ are two positive integers with $k \ll p$. Furthermore, denote by $\mathcal{C}_{k}$ a family of some non-empty subsets of $[p]$ that have cardinality at most $k$. The set $\bigcup_{\mathcal{S} \in \mathcal{C}_{k}} 2^{\mathcal{S}}$ is called a sparsity model of order $k$ generated by $\mathcal{C}_{k}$ and denoted by $\mathcal{M}\left(\mathcal{C}_{k}\right)$

Remark 1. Note that if a set $\mathcal{S} \in \mathcal{C}_{k}$ is a subset of another set in $\mathcal{C}_{k}$, then the same sparsity model can still be generated after removing $\mathcal{S}$ from $\mathcal{C}_{k}$ (i.e., $\mathcal{M}\left(\mathcal{C}_{k}\right)=\mathcal{M}\left(\mathcal{C}_{k} \backslash\{\mathcal{S}\}\right)$ ). Thus, we can assume that there is no pair of distinct sets in $\mathcal{C}_{k}$ that one is a subset of the other.

In this paper we aim to approximate the solution to the optimization problem

$$
\underset{\boldsymbol{\theta} \in \mathcal{H}}{\operatorname{argmin}} f(\boldsymbol{\theta}) \quad \text { s.t. } \operatorname{supp}(\boldsymbol{\theta}) \in \mathcal{M}\left(\mathcal{C}_{k}\right),
$$

where $f: \mathcal{H} \mapsto \mathbb{R}$ is a cost function with $\mathcal{H}$ being a $p$-dimensional real Hilbert space, and $\mathcal{M}\left(\mathcal{C}_{k}\right)$ a given sparsity model described by Def. 1 .

Remark 2. In the context of statistical estimation, the cost function $f(\cdot)$ is usually the empirical loss associated with some observations generated by an underlying true parameter $\theta^{\star}$. In these problems, it is more desired to estimate $\theta^{\star}$ as it describes 


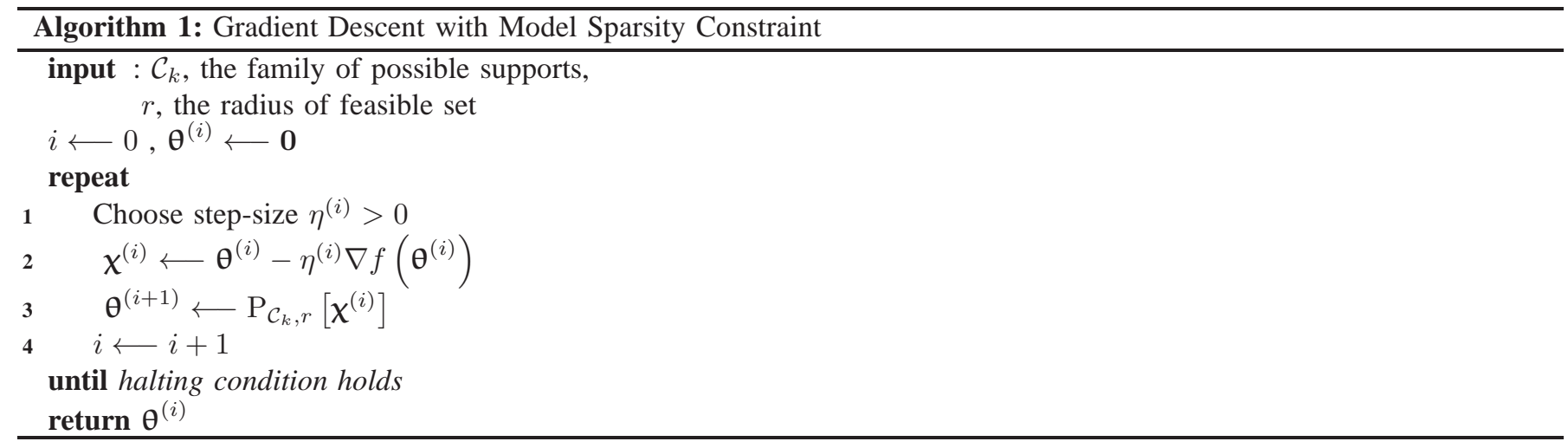

the data. The analysis presented in this paper allows evaluating the approximation error of the proposed algorithm with respect to any parameter vector in the considered sparsity model including any solution $\widehat{\theta}$ to (1) as well as the statistical truth $\theta^{\star}$. However, the approximation error with respect to $\theta^{\star}$ can be simplified and interpreted to a greater extent. We elaborate more on this in Section

To approximate a solution $\widehat{\theta}$ to (1) we use a projected gradient descent method summarized in Algorithm 1 The only difference between Algorithm 1 and standard projected gradient descent methods studied in convex optimization literature is that the projection, in line 3 is performed onto the generally non-convex set $\mathcal{M}\left(\mathcal{C}_{k}\right)$. The projection operator $\mathrm{P}_{\mathcal{C}_{k}, r}: \mathcal{H} \mapsto \mathcal{H}$ at any given point $\theta_{0} \in \mathcal{H}$ is defined as a solution to

$$
\underset{\boldsymbol{\theta} \in \mathcal{H}}{\operatorname{argmin}}\left\|\boldsymbol{\theta}-\theta_{0}\right\| \quad \text { s.t. } \operatorname{supp}(\boldsymbol{\theta}) \in \mathcal{M}\left(\mathcal{C}_{k}\right) \text { and }\|\boldsymbol{\theta}\| \leq r .
$$

Remark 3. One may also question the necessity of the constraint $\|\theta\| \leq r$ in (2). As discussed later in Section [V] in statistical estimation problems where the cost function is not quadratic the sufficient condition we rely on cannot be guaranteed to hold unless the iterates and the true parameter lie in a bounded set. This shortcoming is typical for convergence proofs that use similar types of conditions (cf. [17]-[20]). Finally, the exact projection onto the sparsity model $\mathcal{M}\left(\mathcal{C}_{k}\right)$ might not be tractable.

Existence and complexity of algorithms that find the desired exact or approximate projections, disregarding the length constraint in (2) (i.e., $\mathrm{P}_{\mathcal{C}_{\mathrm{k}},+\infty}[\cdot]$ ), are studied in [7] for several interesting sparsity models. Furthermore, such projections are known and tractable for signals with block-sparse support or support that satisfies a tree model [4]. Often, one may also desire to show that accuracy can be guaranteed even using an inexact projection operator, at the cost of an extra error term. For example, it is recently shown in [21] how to extend the framework of model-based compressed sensing to admit inexact projections by assuming "head" and "tail" oracles for the projections. In other cases, such as the co-sparse analysis signal model, such projections are assumed but not theoretically backed [22]-[24]. Also, in the general case where $r<+\infty$, one can derive a projection $\mathrm{P}_{\mathcal{C}_{k}, r}[\boldsymbol{\theta}]$ from $\mathrm{P}_{\mathcal{C}_{k},+\infty}[\boldsymbol{\theta}]$ (see Lemma 2 in the Appendix).

It is straightforward to generalize the guarantees in this paper to cases where only approximate projection is tractable. However, we do not attempt it here; our focus is to study the algorithm when the cost function is not necessarily quadratic. Instead, we apply the results to statistical estimation problems with non-linear models and we derive bounds on the statistical error of the estimate.

\section{TheOrETICAL ANALYSIS}

\section{A. Stable Model-Restricted Hessian}

In order to demonstrate accuracy of estimates obtained using Algorithm 1 we require a variant of the Stable Restricted Hessian (SRH) condition proposed in [25] to hold. The SRH condition basically characterizes cost functions that have bounded curvature over canonical sparse subspaces. In this paper we require this condition to hold merely for the signals that belong to the considered model. Furthermore, we explicitly bound the length of the vectors at which the condition should hold. As will be discussed later, this restriction is necessary in general for non-quadratic cost functions. The condition we rely on, the Stable Model-Restricted Hessian (SMRH), can be formally defined as follows.

Definition 2. Let $f: \mathcal{H} \mapsto \mathbb{R}$ be a twice continuously differentiable function. Furthermore, let $\alpha_{\mathcal{C}_{k}}$ and $\beta_{\mathcal{C}_{k}}$ be in turn the largest and smallest positive real numbers such that

$$
\beta_{\mathcal{C}_{k}}\|\boldsymbol{\Delta}\|^{2} \leq\left\langle\boldsymbol{\Delta}, \nabla^{2} f(\boldsymbol{\theta}) \boldsymbol{\Delta}\right\rangle \leq \alpha_{\mathcal{C}_{k}}\|\boldsymbol{\Delta}\|^{2},
$$

holds for all $\boldsymbol{\Delta}$ and $\boldsymbol{\theta}$ such that $\operatorname{supp}(\boldsymbol{\Delta}) \cup \operatorname{supp}(\boldsymbol{\theta}) \in \mathcal{M}\left(\mathcal{C}_{k}\right)$ and $\|\boldsymbol{\theta}\| \leq r$. Then $f$ is said to have a Stable Model-Restricted Hessian (SMRH) with respect to the model $\mathcal{M}\left(\mathcal{C}_{k}\right)$ with constants $\alpha_{\mathcal{C}_{k}}$ and $\beta_{\mathcal{C}_{k}}$ in a sphere of radius $r>0$, or in short $\left(\alpha_{\mathcal{C}_{k}}\right.$, $\left.\beta_{\mathcal{C}_{k}}, r\right)$-SMRH. The conditioning for this SMRH is also denoted by $\mu_{\mathcal{C}_{k}}:=\alpha_{\mathcal{C}_{k}} / \beta_{\mathcal{C}_{k}}$. 
Consider the special case of $f(\boldsymbol{\theta})=\frac{1}{2}\|\mathbf{X} \boldsymbol{\theta}-\mathbf{y}\|_{2}^{2}$ as in compressed sensing or sparse linear regression. It is straightforward to see that the SMRH effectively reduces to the model-based restricted isometry property by setting $\nabla^{2} f(\boldsymbol{\theta})=\mathbf{X}^{\top} \mathbf{X}$ in the definition of the SMRH. The model-based restricted isometry constant $\delta_{\mathcal{C}_{k}}$ and the SMRH constants are related in this special case via $\beta_{\mathcal{C}_{k}} \geq 1-\delta_{\mathcal{C}_{k}}, \alpha_{\mathcal{C}_{k}} \leq 1+\delta_{\mathcal{C}_{k}}$, and $\mu_{\mathcal{C}_{k}} \leq\left(1+\delta_{\mathcal{C}_{k}}\right) /\left(1-\delta_{\mathcal{C}_{k}}\right)$.

Remark 4. Typically in parametric estimation problems a sample loss function $\ell(\boldsymbol{\theta}, \mathbf{x}, y)$ is associated with the covariateresponse pair $(\mathbf{x}, y)$ and a parameter $\boldsymbol{\theta}$. Given $n$ iid observations the empirical loss is formulated as $\widehat{L}_{n}(\theta)=$ $\frac{1}{n} \sum_{i=1}^{n} \ell\left(\boldsymbol{\theta}, \mathbf{x}_{i}, y_{i}\right)$. The estimator under study is the minimizer of the empirical loss, perhaps considering an extra regularization or constraint for the parameter $\theta$. To prove accuracy of sparse estimation algorithms it is often required that the cost function is strongly convex/smooth over a restricted set of directions as a sufficient condition. It is known, however, that $\widehat{L}_{n}(\boldsymbol{\theta})$ as an empirical process is a good approximation of the expected loss $L(\boldsymbol{\theta})=\mathbb{E}[\ell(\boldsymbol{\theta}, \mathbf{x}, y)]$ (see [26] and [27, Chapter 5]). If $L(\theta)$ does not satisfy the desired restricted strong convexity/smoothness conditions globally for all choices of the true parameter $\theta^{\star}$ that have the structured sparsity, then in general $\widehat{L}_{n}(\theta)$ does not satisfy the desired conditions globally, either. Thus, as also assumed in the prior work either explicitly [17] or implicitly [18]-[20], for a generic sample loss it is only possible to guarantee these types of sufficient conditions if the set of valid vectors $\theta^{\star}$ are further restricted, e.g., by bounding their length. This is the motivation behind the restriction imposed on the length of $\theta$ in Def. 2 Of course, if the true parameter violates this restriction we may incur an estimation bias as quantified in Theorem 1

The SMRH is similar to other conditions such as SRH [25] and various forms of Restricted Strong Convexity/Smoothness (RSC/RSS) (e.g., [18] and [16]): they all impose quadratic bounds on the second derivative of the objective function when restricted to sparse or model-sparse vectors. However, there are some subtle differences. The SRH is defined for plain sparse vectors and its quadratic bounds are defined locally. For the SMRH, however, the fact that the boundedness is incorporated in the signal model allowed us to define the quadratic bounds globally. The RSC defined in [16] is more general than the SMRH since it assumes infinite-dimensional Hilbert spaces as the domain of the function, whereas in SMRH we consider function defined over finite-dimensional Hilbert spaces. However, the accuracy analysis of [16] guarantees convergence of the projected gradient descent $\mu_{\mathcal{C}_{k}^{3}}<4 / 3$, whereas, as will be shown by Corollary 1 , we can prove convergence of the algorithm for $\mu_{\mathcal{C}_{k}^{3}}<3 / 2$ or even $\mu_{\mathcal{C}_{k}^{3}}<3$.

\section{B. Accuracy Guarantee}

Recall that in our notation $\mathcal{C}_{k}^{2}=\mathcal{C}_{k} \uplus \mathcal{C}_{k}$ and $\mathcal{C}_{k}^{3}=\mathcal{C}_{k} \uplus \mathcal{C}_{k} \uplus \mathcal{C}_{k}$. Intuitively, $\mathcal{C}_{k}^{2}$ and $\mathcal{C}_{k}^{3}$ can describe all possible support sets of the sum of two or three vectors in $\mathcal{M}\left(\mathcal{C}_{k}\right)$, respectively. Using the notion of SMRH we can now state the main theorem.

Theorem 1. Consider the sparsity model $\mathcal{M}\left(\mathcal{C}_{k}\right)$ for some $k \in \mathbb{N}$ and a cost function $f: \mathcal{H} \mapsto \mathbb{R}$ that satisfies the $\left(\alpha_{\mathcal{C}_{k}^{3}}, \beta_{\mathcal{C}_{k}^{3}}, r\right)$-SMRH condition as in (3) with $\mu_{\mathcal{C}_{k}^{3}}:=\alpha_{\mathcal{C}_{k}^{3}} / \beta_{\mathcal{C}_{k}^{3}}$. If $\eta^{\star}=2 /\left(\alpha_{\mathcal{C}_{k}^{3}}+\beta_{\mathcal{C}_{k}^{3}}\right)$ then for any $\overline{\boldsymbol{\theta}} \in \mathcal{M}\left(\mathcal{C}_{k}\right)$ with $\|\bar{\theta}\| \leq r$ the iterates of Algorithm $\square$ obey

$$
\left\|\boldsymbol{\theta}^{(i+1)}-\overline{\boldsymbol{\theta}}\right\| \leq 2 \gamma^{(i)}\left\|\boldsymbol{\theta}^{(i)}-\overline{\boldsymbol{\theta}}\right\|+2 \eta^{(i)}\left\|\nabla_{\overline{\mathcal{I}}} f(\overline{\boldsymbol{\theta}})\right\|,
$$

where $\gamma^{(i)}=\frac{\eta^{(i)}}{\eta^{\star}} \frac{\mu_{\mathcal{C}_{k}^{3}}-1}{\mu_{\mathcal{C}_{k}^{3}}+1}+\left|\frac{\eta^{(i)}}{\eta^{\star}}-1\right|$ and $\overline{\mathcal{I}}=\operatorname{supp}\left(\mathrm{P}_{\mathcal{C}_{k}^{2}, r}[\nabla f(\bar{\theta})]\right)$.

Theorem 1 can be used to localize an "attractor set" of the iterates $\theta^{(i)}$ with respect to the desired reference point $\bar{\theta}$. In particular, if $2 \gamma^{(i)}<1$ the bound (4) guarantees an approximate contraction which can be used recursively as in Corollary 1 below. Ideally, the iterates eventually fall within a relatively small ball centered at the desired $\bar{\theta}$. We refer to the radius of this ball as the approximation error. Theorem 1 helps to bound the approximation error in terms of $\nabla f(\bar{\theta})$. For instance, if at a model-sparse minimizer obtained by (1) the gradient of the objective (restricted to indices $\overline{\mathcal{I}}$ ) has a small $\ell_{2}$-norm then the iterates can provide accurate estimates of the minimizer. In particular, if the restricted gradient vanishes at a model-sparse minimizer, the approximation error with respect to that point would be zero, i.e., the iterates converge provided that $2 \gamma^{(i)}<1$ is guaranteed. This scenario can occur when the objective has multiple stationary points only one of which is within the model, a typical case in high-dimensional estimation problems. For example, classical guarantees for noiseless sparse linear regression provide exact recovery if the signal is exactly sparse, but only an approximation if the signal is approximately sparse. While (4) still holds if the gradient is not restricted to $\overline{\mathcal{I}}$, the obtained approximation error might not be sufficiently tight. For example, applying (4) in statistical estimation problems with $\nabla f(\theta)$ replacing $\nabla_{\overline{\mathcal{I}}} f(\overline{\boldsymbol{\theta}})$ would yield loose estimation error bounds that grow with the ambient dimension rather than the sparsity of the target parameter.

Remark 5. One should choose the step size to achieve a contraction factor $2 \gamma^{(i)}$ that is as small as possible. Straightforward algebra shows that the constant step-size $\eta^{(i)}=\eta^{\star}$ is optimal, but this choice may not be practical as the constants $\alpha_{\mathcal{C}_{k}^{3}}$ and $\beta_{\mathcal{C}_{k}^{3}}$ might not be known. Instead, we can always choose the step-size such that $1 / \alpha_{\mathcal{C}_{k}^{3}} \leq \eta^{(i)} \leq 1 / \beta_{\mathcal{C}_{k}^{3}}$ provided that the cost function obeys the SMRH condition. It suffices to set $\eta^{(i)}=1 /\left\langle\boldsymbol{\Delta}, \nabla^{2} f(\boldsymbol{\theta}) \boldsymbol{\Delta}\right\rangle$ for some $\boldsymbol{\Delta}, \boldsymbol{\theta} \in \mathcal{H}$ that obeys $\operatorname{supp}(\boldsymbol{\Delta}) \cup \operatorname{supp}(\boldsymbol{\theta}) \in \mathcal{M}\left(\mathcal{C}_{k}^{3}\right)$. For this choice of $\eta^{(i)}$, we have $\gamma^{(i)} \leq \mu_{\mathcal{C}_{k}^{3}}-1$. 
Corollary 1. A fixed step-size $\eta>0$ corresponds to a fixed contraction coefficient $\gamma=\frac{\eta}{\eta^{\star}} \frac{\mu_{\mathcal{C}_{k}^{3}}-1}{\mu_{\mathcal{C}_{k}^{3}}+1}+\left|\frac{\eta}{\eta^{\star}}-1\right|$. In this case, assuming that $2 \gamma \neq 1$, the $i$-th iterate of Algorithm $\square$ satisfies

$$
\left\|\boldsymbol{\theta}^{(i)}-\overline{\boldsymbol{\theta}}\right\| \leq(2 \gamma)^{i}\|\overline{\boldsymbol{\theta}}\|+2 \eta \frac{1-(2 \gamma)^{i}}{1-2 \gamma}\left\|\nabla_{\overline{\mathcal{I}}} f(\overline{\boldsymbol{\theta}})\right\|
$$

In particular,

(i) if $\mu_{\mathcal{C}_{k}^{3}}<3$ and $\eta=\eta^{\star}=2 /\left(\alpha_{\mathcal{C}_{k}^{3}}+\beta_{\mathcal{C}_{k}^{3}}\right)$, or

(ii) if $\mu_{\mathcal{C}_{k}^{3}}<\frac{3}{2}$ and $\eta \in\left[1 / \alpha_{\mathcal{C}_{k}^{3}}, 1 / \beta_{\mathcal{C}_{k}^{3}}\right]$,

the distance of the iterates and $\bar{\theta}$ shrinks up to an approximation error bounded above by $\frac{2 \eta}{1-2 \gamma}\left\|\nabla_{\overline{\mathcal{I}}} f(\bar{\theta})\right\|$ with contraction factor $2 \gamma<1$.

Proof: Applying (4) recursively under the assumptions of the corollary and using the identity $\sum_{j=0}^{i-1}(2 \gamma)^{j}=\frac{1-(2 \gamma)^{i}}{1-2 \gamma}$ proves (5). In the first case, if $\mu_{\mathcal{C}_{k}^{3}}<3$ and $\eta=\eta^{\star}=2 /\left(\alpha_{\mathcal{C}_{k}^{3}}+\beta_{\mathcal{C}_{k}^{3}}\right)$ we have $2 \gamma<1$ by definition of $\gamma$. In the second case, one can deduce from $\eta \in\left[1 / \alpha_{\mathcal{C}_{k}^{3}}, 1 / \beta_{\mathcal{C}_{k}^{3}}\right]$ that $\left|\eta / \eta^{\star}-1\right| \leq \frac{\mu_{\mathcal{C}_{k}^{3}}-1}{2}$ and $\eta / \eta^{\star} \leq \frac{\mu_{\mathcal{C}_{k}^{3}}+1}{2}$ where equalities are attained simultaneously at $\eta=1 / \beta_{\mathcal{C}_{k}^{3}}$. Therefore, $\gamma \leq \mu_{\mathcal{C}_{k}^{3}}-1<1 / 2$ and thus $2 \gamma<1$. Finally, in both cases it immediately follows from (5) that the approximation error converges to $\frac{2 \eta}{1-2 \gamma}\left\|\nabla_{\overline{\mathcal{I}}} f(\bar{\theta})\right\|$ from below as $i \rightarrow+\infty$.

\section{Application in Generalized Linear Models}

Generalized Linear Models (GLMs) are among the most commonly used models for parametric estimation in variety of applications [28]. Linear, logistic, Poisson, and gamma models used in corresponding regression problems all belong to the family of GLMs. Given a covariate vector $\mathbf{x} \in \mathcal{X} \subseteq \mathbb{R}^{p}$ and a true parameter $\boldsymbol{\theta}^{\star} \in \mathbb{R}^{p}$, the response variable $y \in \mathcal{Y} \subseteq \mathbb{R}$ in canonical GLMs is assumed to follow an exponential family conditional distribution: $y \mid \mathbf{x} ; \boldsymbol{\theta}^{\star} \sim Z(y) \exp \left(y\left\langle\mathbf{x}, \boldsymbol{\theta}^{\star}\right\rangle-\psi\left(\left\langle\mathbf{x}, \boldsymbol{\theta}^{\star}\right\rangle\right)\right)$, where $Z(y)$ is a positive function, and $\psi: \mathbb{R} \mapsto \mathbb{R}$ is the log-partition function that satisfies $\psi(t)=\log \int_{\mathcal{Y}} Z(y) \exp (t y) \mathrm{d} y$ for all $t \in \mathbb{R}$. Examples of the log-partition function include but are not limited to $\psi_{\operatorname{lin}}(t)=t^{2} / 2 \sigma^{2}, \psi_{\log }(t)=\log (1+\exp (t))$, and $\psi_{\text {Pois }}(t)=\exp (t)$ corresponding to linear, logistic, and Poisson models, respectively.

Suppose that $n$ iid covariate-response pairs $\left\{\left(\mathbf{x}_{i}, y_{i}\right)\right\}_{i=1}^{n}$ are observed. In the Maximum Likelihood Estimation (MLE) framework the negative log likelihood is used as a measure of the discrepancy between the true parameter $\theta^{\star}$ and an estimate $\theta$ based on the observations. Formally, the average of negative log likelihoods is considered as the empirical loss

$$
f(\boldsymbol{\theta})=\frac{1}{n} \sum_{i=1}^{n} \psi\left(\left\langle\mathbf{x}_{i}, \boldsymbol{\theta}\right\rangle\right)-y_{i}\left\langle\mathbf{x}_{i}, \boldsymbol{\theta}\right\rangle,
$$

and the MLE is performed by minimizing $f(\theta)$ over the set of feasible $\theta$. The constants $c$ and $Z$ that appear in the distribution are disregarded as they have no effect in the outcome.

\section{A. Verifying SMRH for GLMS}

Assuming that $\psi(\cdot)$ is twice continuously differentiable, the Hessian of $f(\cdot)$ is equal to

$$
\nabla^{2} f(\boldsymbol{\theta})=\frac{1}{n} \sum_{i=1}^{n} \psi^{\prime \prime}\left(\left\langle\mathbf{x}_{i}, \boldsymbol{\theta}\right\rangle\right) \mathbf{x}_{i} \mathbf{x}_{i}^{\top} .
$$

Under the assumptions for GLMs, it can be shown that $\psi^{\prime \prime}(\cdot)$ is non-negative (i.e., $\psi(\cdot)$ is convex). For a given sparsity model generated by $\mathcal{C}_{k}$ let $\mathcal{S}$ be an arbitrary support set in $\mathcal{C}_{k}$ and suppose that $\operatorname{supp}(\theta) \subseteq \mathcal{S}$ and $\|\theta\| \leq r$. Furthermore, define

$$
D_{\psi, r}(u):=\max _{t \in[-r, r]} \psi^{\prime \prime}(t u) \quad \text { and } \quad d_{\psi, r}(u):=\min _{t \in[-r, r]} \psi^{\prime \prime}(t u) .
$$

Using the Cauchy-Schwarz inequality we have $\left|\left\langle\mathbf{x}_{i}, \boldsymbol{\theta}\right\rangle\right| \leq r\left\|\left.\mathbf{x}_{i}\right|_{\mathcal{S}}\right\|$ which implies

$$
\left.\left.\left.\left.\frac{1}{n} \sum_{i=1}^{n} d_{\psi, r}\left(\left\|\left.\mathbf{x}_{i}\right|_{\mathcal{S}}\right\|\right) \mathbf{x}_{i}\right|_{\mathcal{S}} \mathbf{x}_{i}\right|_{\mathcal{S}} ^{\top} \preccurlyeq \nabla_{\mathcal{S}}^{2} f(\boldsymbol{\theta}) \preccurlyeq \frac{1}{n} \sum_{i=1}^{n} D_{\psi, r}\left(\left\|\left.\mathbf{x}_{i}\right|_{\mathcal{S}}\right\|\right) \mathbf{x}_{i}\right|_{\mathcal{S}} \mathbf{x}_{i}\right|_{\mathcal{S}} ^{\top} .
$$

These matrix inequalities are precursors of (3). Imposing further restriction on the distribution of the covariate vectors $\left\{\mathbf{x}_{i}\right\}_{i=1}^{n}$ allows application of the results from random matrix theory regarding the extreme eigenvalues of random matrices (see e.g., [29] and [30]).

For example, in the logistic model where $\psi \equiv \psi_{\log }$ we can show that $D_{\psi, r}(u)=\frac{1}{4}$ and $d_{\psi, r}(u)=\frac{1}{4} \operatorname{sech}^{2}\left(\frac{r u}{2}\right)$. Assuming that the covariate vectors are iid instances of a random vectors whose length almost surely bounded by one, we obtain $d_{\psi, r}(u) \geq \frac{1}{4} \operatorname{sech}^{2}\left(\frac{r}{2}\right)$. Using the matrix Chernoff inequality [29] the extreme eigenvalues of $\frac{1}{n} \mathbf{X}_{\mathcal{S}} \mathbf{X}_{\mathcal{S}}^{\top}$ can be bounded with probability $1-\exp (\log k-C n)$ for some constant $C>0$ (see [25] for detailed derivations). Using these results and taking the union bound over all $\mathcal{S} \in \mathcal{C}_{k}$ we obtain bounds for the extreme eigenvalues of $\nabla_{\mathcal{S}}^{2} f(\theta)$ that hold uniformly for all sets $\mathcal{S} \in \mathcal{C}_{k}$ with probability $1-\exp \left(\log \left(k\left|\mathcal{C}_{k}\right|\right)-C n\right)$. Thus (3) may hold if $n=O\left(\log \left(k\left|\mathcal{C}_{k}\right|\right)\right)$. 


\section{B. Approximation Error for GLMs}

Suppose that the approximation error is measured with respect to $\boldsymbol{\theta}^{\perp}=\mathrm{P}_{\mathcal{C}_{k}, r}\left[\boldsymbol{\theta}^{\star}\right]$ where $\boldsymbol{\theta}^{\star}$ is the statistical truth in the considered GLM. It is desirable to further simplify the approximation error bound provided in Corollary 1 which is related to the statistical precision of the estimation problem. The corollary provides an approximation error that is proportional to $\left\|\nabla_{\mathcal{T}} f\left(\boldsymbol{\theta}^{\perp}\right)\right\|$ where $\mathcal{T}=\operatorname{supp}\left(\mathrm{P}_{\mathcal{C}_{k}^{2}, r}\left[\nabla f\left(\theta^{\perp}\right)\right]\right)$. We can write

$$
\nabla_{\mathcal{T}} f\left(\boldsymbol{\theta}^{\perp}\right)=\left.\frac{1}{n} \sum_{i=1}^{n}\left(\psi^{\prime}\left(\left\langle\mathbf{x}_{i}, \boldsymbol{\theta}^{\perp}\right\rangle\right)-y_{i}\right) \mathbf{x}_{i}\right|_{\mathcal{T}}
$$

which yields $\left\|\nabla_{\mathcal{T}} f\left(\boldsymbol{\theta}^{\perp}\right)\right\|=\left\|\mathbf{X}_{\mathcal{T}} \mathbf{z}\right\|$ where $\mathbf{X}=\frac{1}{\sqrt{n}}\left[\begin{array}{llll}\mathbf{x}_{1} & \mathbf{x}_{2} & \cdots & \mathbf{x}_{n}\end{array}\right]$ and $\left.\mathbf{z}\right|_{\{i\}}=z_{i}=\frac{\psi^{\prime}\left(\left\langle\mathbf{x}_{i}, \boldsymbol{\theta}^{\perp}\right\rangle\right)-y_{i}}{\sqrt{n}}$. Therefore,

$$
\left\|\nabla_{\mathcal{T}} f\left(\boldsymbol{\theta}^{\perp}\right)\right\|^{2} \leq\left\|\mathbf{X}_{\mathcal{T}}\right\|_{\text {op }}^{2}\|\mathbf{z}\|^{2}
$$

where $\|\cdot\|_{\mathrm{op}}$ denotes the operator norm. Again using random matrix theory one can find an upper bound for $\left\|\mathbf{X}_{\mathcal{I}}\right\|_{\mathrm{op}}$ that holds uniformly for any $\mathcal{I} \in \mathcal{C}_{k}^{2}$ and in particular for $\mathcal{I}=\mathcal{T}$. Henceforth, $W>0$ is used to denote this upper bound.

The second term in the bound can be written as

$$
\|\mathbf{z}\|^{2}=\frac{1}{n} \sum_{i=1}^{n}\left(\psi^{\prime}\left(\left\langle\mathbf{x}_{i}, \boldsymbol{\theta}^{\perp}\right\rangle\right)-y_{i}\right)^{2}
$$

To further simplify this term we need to make assumptions about the log-partition function $\psi(\cdot)$ and/or the distribution of the covariate-response pair $(\mathbf{x}, y)$. For instance, if $\psi^{\prime}(\cdot)$ and the response variable $y$ are bounded, as in the logistic model, then Hoeffding's inequality implies that for some small $\epsilon>0$ we have $\|\mathbf{z}\|^{2} \leq \mathbb{E}\left[\left(\psi^{\prime}\left(\left\langle\mathbf{x}, \theta^{\perp}\right\rangle\right)-y\right)^{2}\right]+\epsilon$ with probability at least $1-\exp \left(-O\left(\epsilon^{2} n\right)\right)$. Since in GLMs the true parameter $\boldsymbol{\theta}^{\star}$ is the minimizer of the expected loss $\mathbb{E}[\psi(\langle\mathbf{x}, \boldsymbol{\theta}\rangle)-y\langle\mathbf{x}, \boldsymbol{\theta}\rangle \mid \mathbf{x}]$ we deduce that $\mathbb{E}\left[\psi^{\prime}\left(\left\langle\mathbf{x}, \boldsymbol{\theta}^{\star}\right\rangle\right)-y \mid \mathbf{x}\right]=0$ and hence $\mathbb{E}\left[\psi^{\prime}\left(\left\langle\mathbf{x}, \boldsymbol{\theta}^{\star}\right\rangle\right)-y\right]=0$. Therefore,

$$
\begin{aligned}
\|\mathbf{z}\|^{2} & \leq \mathbb{E}\left[\mathbb{E}\left[\left(\psi^{\prime}\left(\left\langle\mathbf{x}, \boldsymbol{\theta}^{\perp}\right\rangle\right)-\psi^{\prime}\left(\left\langle\mathbf{x}, \theta^{\star}\right\rangle\right)+\psi^{\prime}\left(\left\langle\mathbf{x}, \theta^{\star}\right\rangle\right)-y\right)^{2} \mid \mathbf{x}\right]\right]+\epsilon \\
& \leq \mathbb{E}\left[\left(\psi^{\prime}\left(\left\langle\mathbf{x}, \theta^{\perp}\right\rangle\right)-\psi^{\prime}\left(\left\langle\mathbf{x}, \boldsymbol{\theta}^{\star}\right\rangle\right)\right)^{2}\right]++\mathbb{E}\left[\left(\psi^{\prime}\left(\left\langle\mathbf{x}, \theta^{\star}\right\rangle\right)-y\right)^{2}\right]+\epsilon . \\
& =\underbrace{\mathbb{E}\left[\left(\psi^{\prime}\left(\left\langle\mathbf{x}, \theta^{\perp}\right\rangle\right)-\psi^{\prime}\left(\left\langle\mathbf{x}, \boldsymbol{\theta}^{\star}\right\rangle\right)\right)^{2}\right]}_{\delta_{1}}+\underbrace{\operatorname{var}\left(\psi^{\prime}\left(\left\langle\mathbf{x}, \theta^{\star}\right\rangle\right)-y\right)+\epsilon}_{\sigma_{\text {stat }}^{\text {2 }}} .
\end{aligned}
$$

Then it follows from Corollary 1 and the fact that $\left\|\left.\mathbf{X}\right|_{\mathcal{I}}\right\|_{\mathrm{op}} \leq W$ that

$$
\begin{aligned}
\left\|\theta^{(i)}-\theta^{\star}\right\| & \leq\left\|\theta^{(i)}-\theta^{\perp}\right\|+\underbrace{\left\|\theta^{\perp}-\theta^{\star}\right\|}_{\delta_{2}} \\
& \leq(2 \gamma)^{i}\left\|\theta^{\perp}\right\|+\frac{2 \eta W}{1-2 \gamma} \sigma_{\text {stat }}^{2}+\frac{2 \eta W}{1-2 \gamma} \delta_{1}+\delta_{2} .
\end{aligned}
$$

We see the total approximation error is comprised of two parts. The first part is due to statistical error that is given by $\frac{2 \eta W}{1-2 \gamma} \sigma_{\text {stat }}^{2}$, and $\frac{2 \eta W}{1-2 \gamma} \delta_{1}+\delta_{2}$ is the second part of the error due to the bias that occurs because of an infeasible true parameter. The bias vanishes if the true parameter lies in the considered bounded sparsity model (i.e., $\boldsymbol{\theta}^{\star}=\mathrm{P}_{\mathcal{C}_{k}, r}\left[\theta^{\star}\right]$ ).

\section{CONCLUSiON}

We studied the projected gradient descent method for minimization of a real valued cost function defined over a finitedimensional Hilbert space, under structured sparsity constraints. Using previously known combinatorial sparsity models, we define a sufficient condition for accuracy of the algorithm, the SMRH. Under this condition the algorithm produces an approximation for the desired optimum at a linear rate. Unlike the previous results on greedy-type methods that merely have focused on linear statistical models, our algorithm applies to a broader family of estimation problems. To provide an example, we examined application of the algorithm in estimation with GLMs and showed how the SMRH can be verified for these models. The approximation error can also be bounded by statistical precision and the potential bias. An interesting follow-up problem is to find whether the approximation error can be improved and the derived error is merely a by-product of requiring some form of restricted strong convexity through SMRH. 
APPENDIX

PROOFS

Lemma 1. Suppose that $f$ is a twice differentiable function that satisfies (3) for a given $\boldsymbol{\theta}$ and all $\boldsymbol{\Delta}$ such that $\operatorname{supp}(\boldsymbol{\Delta}) \cup$ $\operatorname{supp}(\theta) \in \mathcal{M}\left(\mathcal{C}_{k}\right)$. Then we have

$$
\left|\langle\mathbf{u}, \mathbf{v}\rangle-\eta\left\langle\mathbf{u}, \nabla^{2} f(\boldsymbol{\theta}) \mathbf{v}\right\rangle\right| \leq\left(\eta \frac{\alpha_{\mathcal{C}_{k}}-\beta_{\mathcal{C}_{k}}}{2}+\left|\eta \frac{\alpha_{\mathcal{C}_{k}}+\beta_{\mathcal{C}_{k}}}{2}-1\right|\right)\|\mathbf{u}\|\|\mathbf{v}\|
$$

for all $\eta>0$ and $\mathbf{u}, \mathbf{v} \in \mathcal{H}$ such that $\operatorname{supp}(\mathbf{u} \pm \mathbf{v}) \cup \operatorname{supp}(\boldsymbol{\theta}) \in \mathcal{M}\left(\mathcal{C}_{k}\right)$.

Proof: We first the prove the lemma for unit-norm vectors $\mathbf{u}$ and $\mathbf{v}$. Since $\operatorname{supp}(\mathbf{u} \pm \mathbf{v}) \cup \operatorname{supp}(\boldsymbol{\theta}) \in \mathcal{M}\left(\mathcal{C}_{k}\right)$ we can use (3) for $\Delta=\mathbf{u} \pm \mathbf{v}$ to obtain

$$
\beta_{\mathcal{C}_{k}}\|\mathbf{u} \pm \mathbf{v}\|^{2} \leq\left\langle\mathbf{u} \pm \mathbf{v}, \nabla^{2} f(\boldsymbol{\theta})(\mathbf{u} \pm \mathbf{v})\right\rangle \leq \alpha_{\mathcal{C}_{k}}\|\mathbf{u} \pm \mathbf{v}\|^{2} .
$$

These inequalities and the assumption $\|\mathbf{u}\|=\|\mathbf{v}\|=1$ then yield

$$
\frac{\beta_{\mathcal{C}_{k}}-\alpha_{\mathcal{C}_{k}}}{2}+\frac{\alpha_{\mathcal{C}_{k}}+\beta_{\mathcal{C}_{k}}}{2}\langle\mathbf{u}, \mathbf{v}\rangle \leq\left\langle\mathbf{u}, \nabla^{2} f(\boldsymbol{\theta}) \mathbf{v}\right\rangle \leq \frac{\alpha_{\mathcal{C}_{k}}-\beta_{\mathcal{C}_{k}}}{2}+\frac{\alpha_{\mathcal{C}_{k}}+\beta_{\mathcal{C}_{k}}}{2}\langle\mathbf{u}, \mathbf{v}\rangle,
$$

where we used the fact that $\nabla^{2} f(\theta)$ is symmetric since $f$ is twice continuously differentiable. Multiplying all sides by $\eta$ and rearranging the terms then imply

$$
\begin{aligned}
\eta \frac{\alpha_{\mathcal{C}_{k}}-\beta_{\mathcal{C}_{k}}}{2} & \geq\left|\left(\eta \frac{\alpha_{\mathcal{C}_{k}}+\beta_{\mathcal{C}_{k}}}{2}-1\right)\langle\mathbf{u}, \mathbf{v}\rangle+\langle\mathbf{u}, \mathbf{v}\rangle-\eta\left\langle\mathbf{u}, \nabla^{2} f(\boldsymbol{\theta}) \mathbf{v}\right\rangle\right| \\
& \geq\left|\langle\mathbf{u}, \mathbf{v}\rangle-\eta\left\langle\mathbf{u}, \nabla^{2} f(\boldsymbol{\theta}) \mathbf{v}\right\rangle\right|-\left|\left(\eta \frac{\alpha_{\mathcal{C}_{k}}+\beta_{\mathcal{C}_{k}}}{2}-1\right)\langle\mathbf{u}, \mathbf{v}\rangle\right| \\
& \geq\left|\langle\mathbf{u}, \mathbf{v}\rangle-\eta\left\langle\mathbf{u}, \nabla^{2} f(\boldsymbol{\theta}) \mathbf{v}\right\rangle\right|-\left|\eta \frac{\alpha_{\mathcal{C}_{k}}+\beta_{\mathcal{C}_{k}}}{2}-1\right|
\end{aligned}
$$

which is equivalent to result for unit-norm $\mathbf{u}$ and $\mathbf{v}$ as desired. For the general case one can write $\mathbf{u}=\|\mathbf{u}\| \mathbf{u}^{\prime}$ and $\mathbf{v}=\|\mathbf{v}\| \mathbf{v}^{\prime}$ such that $\mathbf{u}^{\prime}$ and $\mathbf{v}^{\prime}$ are both unit-norm. It is straightforward to verify that using (6) for $\mathbf{u}^{\prime}$ and $\mathbf{v}^{\prime}$ as the unit-norm vectors and multiplying both sides of the resulting inequality by $\|\mathbf{u}\|\|\mathbf{v}\|$ yields the desired general case.

Proof of Theorem [1 U Using optimality of $\theta^{(i+1)}$ and feasibility of $\bar{\theta}$ one can deduce $\left\|\theta^{(i+1)}-\chi^{(i)}\right\|^{2} \leq\left\|\bar{\theta}-\chi^{(i)}\right\|^{2}$, with $\chi^{(i)}$ as in line 2 of Algorithm 1 Expanding the squared norms using the inner product of $\mathcal{H}$ then shows $0 \leq$ $\left\langle\boldsymbol{\theta}^{(i+1)}-\overline{\boldsymbol{\theta}}, 2 \boldsymbol{\chi}^{(i)}-\boldsymbol{\theta}^{(i+1)}-\overline{\boldsymbol{\theta}}\right\rangle$ or equivalently $0 \leq\left\langle\boldsymbol{\Delta}^{(i+1)}, 2 \boldsymbol{\theta}^{(i)}-2 \eta^{(i)} \nabla f\left(\overline{\boldsymbol{\theta}}+\boldsymbol{\Delta}^{(i)}\right)-\boldsymbol{\Delta}^{(i+1)}\right\rangle$, where $\boldsymbol{\Delta}^{(i)}=\boldsymbol{\theta}^{(i)}-\overline{\bar{\theta}}$ and $\boldsymbol{\Delta}^{(i+1)}=\theta^{(i+1)}-\overline{\boldsymbol{\theta}}$. Adding and subtracting $2 \eta^{(i)}\left\langle\boldsymbol{\Delta}^{(i+1)}, \nabla f(\overline{\boldsymbol{\theta}})\right\rangle$ and rearranging yields

$$
\begin{aligned}
\left\|\boldsymbol{\Delta}^{(i+1)}\right\|^{2} & \leq 2\left\langle\boldsymbol{\Delta}^{(i+1)}, \boldsymbol{\theta}^{(i)}\right\rangle-2 \eta^{(i)}\left\langle\boldsymbol{\Delta}^{(i+1)}, \nabla f\left(\overline{\boldsymbol{\theta}}+\boldsymbol{\Delta}^{(i)}\right)-\nabla f(\overline{\boldsymbol{\theta}})\right\rangle \\
& -2 \eta^{(i)}\left\langle\boldsymbol{\Delta}^{(i+1)}, \nabla f(\overline{\boldsymbol{\theta}})\right\rangle
\end{aligned}
$$

Since $f$ is twice continuously differentiable by assumption, it follows form the mean-value theorem that $\left\langle\boldsymbol{\Delta}^{(i+1)}, \nabla f\left(\overline{\boldsymbol{\theta}}+\boldsymbol{\Delta}^{(i)}\right)-\nabla f(\overline{\boldsymbol{\theta}})\right\rangle=\left\langle\boldsymbol{\Delta}^{(i+1)}, \nabla^{2} f\left(\overline{\boldsymbol{\theta}}+t \boldsymbol{\Delta}^{(i)}\right) \boldsymbol{\Delta}^{(i)}\right\rangle$, for some $t \in(0,1)$. Furthermore, because $\overline{\boldsymbol{\theta}}$, $\boldsymbol{\theta}^{(i)}, \boldsymbol{\theta}^{(i+1)}$ all belong to the model set $\mathcal{M}\left(\mathcal{C}_{k}\right)$ we have $\operatorname{supp}\left(\overline{\boldsymbol{\theta}}+t \boldsymbol{\Delta}^{(i)}\right) \in \mathcal{M}\left(\mathcal{C}_{k}^{2}\right)$ and thereby $\operatorname{supp}\left(\boldsymbol{\Delta}^{(i+1)}\right) \cup$ $\operatorname{supp}\left(\overline{\boldsymbol{\theta}}+t \boldsymbol{\Delta}^{(i)}\right) \in \mathcal{M}\left(\mathcal{C}_{k}^{3}\right)$. Invoking the $\left(\alpha_{\mathcal{C}_{k}^{3}}, \beta_{\mathcal{C}_{k}^{3}}, r\right)$-SMRH condition of the cost function and applying Lemma 1 with the sparsity model $\mathcal{M}\left(\mathcal{C}_{k}^{3}\right), \theta=\bar{\theta}+t \boldsymbol{\Delta}^{(i)}$, and $\eta=\eta^{(i)}$ then yields

$$
\left|\left\langle\boldsymbol{\Delta}^{(i+1)}, \boldsymbol{\Delta}^{(i)}\right\rangle-\eta^{(i)}\left\langle\boldsymbol{\Delta}^{(i+1)}, \nabla f\left(\overline{\boldsymbol{\theta}}+\boldsymbol{\Delta}^{(i)}\right)-\nabla f(\overline{\boldsymbol{\theta}})\right\rangle\right| \leq \gamma^{(i)}\left\|\boldsymbol{\Delta}^{(i+1)}\right\|\left\|\boldsymbol{\Delta}^{(i)}\right\| .
$$

Using the Cauchy-Schwarz inequality and the fact that $\left\|\nabla_{\operatorname{supp}\left(\Delta^{(i+1)}\right)} f(\overline{\boldsymbol{\theta}})\right\| \leq\left\|\nabla_{\overline{\mathcal{I}}} f(\overline{\boldsymbol{\theta}})\right\|$ by the definition of $\overline{\mathcal{I}}$, (7) implies that $\left\|\boldsymbol{\Delta}^{(i+1)}\right\|^{2} \leq 2 \gamma^{(i)}\left\|\boldsymbol{\Delta}^{(i+1)}\right\|\left\|\boldsymbol{\Delta}^{(i)}\right\|+2 \eta^{(i)}\left\|\boldsymbol{\Delta}^{(i+1)}\right\|\left\|\nabla_{\overline{\mathcal{I}}} f(\overline{\boldsymbol{\theta}})\right\|$. Canceling $\left\|\boldsymbol{\Delta}^{(i+1)}\right\|$ from both sides proves the theorem.

Lemma 2 (Bounded Model Projection). Given an arbitrary $\mathbf{h}_{0} \in \mathcal{H}$, a positive real number $r$, and a sparsity model generator $\mathcal{C}_{k}$, a projection $\mathrm{P}_{\mathcal{C}_{k}, r}\left[\mathbf{h}_{0}\right]$ can be obtained as the projection of $\mathrm{P}_{\mathcal{C}_{k},+\infty}\left[\mathbf{h}_{0}\right]$ on to the sphere of radius $r$.

Proof: To simplify the notation let $\widehat{\mathbf{h}}=\mathrm{P}_{\mathcal{C}_{k}, r}\left[\mathbf{h}_{0}\right]$ and $\widehat{\mathcal{S}}=\operatorname{supp}(\widehat{\mathbf{h}})$. For $\mathcal{S} \subseteq[p]$ define

$$
\mathbf{h}_{0}(\mathcal{S})=\underset{\mathbf{h}}{\operatorname{argmin}}\left\|\mathbf{h}-\mathbf{h}_{0}\right\| \quad \text { s.t. }\|\mathbf{h}\| \leq r \text { and } \operatorname{supp}(\mathbf{h}) \subseteq \mathcal{S} .
$$


It follows from the definition of $\mathrm{P}_{\mathcal{C}_{k}, r}\left[\mathbf{h}_{0}\right]$ that $\widehat{\mathcal{S}} \in \operatorname{argmin}_{\mathcal{S} \in \mathcal{C}_{k}}\left\|\mathbf{h}_{0}(\mathcal{S})-\mathbf{h}_{0}\right\|$. Using

$$
\left\|\mathbf{h}_{0}(\mathcal{S})-\mathbf{h}_{0}\right\|^{2}=\left\|\mathbf{h}_{0}(\mathcal{S})-\left.\mathbf{h}_{0}\right|_{\mathcal{S}}-\left.\mathbf{h}_{0}\right|_{\mathcal{S}^{c}}\right\|^{2}=\left\|\mathbf{h}_{0}(\mathcal{S})-\left.\mathbf{h}_{0}\right|_{\mathcal{S}}\right\|^{2}+\left\|\left.\mathbf{h}_{0}\right|_{\mathcal{S}^{c}}\right\|^{2},
$$

we deduce that $\mathbf{h}_{0}(\mathcal{S})$ is the projection of $\left.\mathbf{h}_{0}\right|_{\mathcal{S}}$ onto the sphere of radius $r$. Therefore, we can write $\mathbf{h}_{0}(\mathcal{S})=$ $\left.\min \left\{1, r /\left\|\left.\mathbf{h}_{0}\right|_{\mathcal{S}}\right\|\right\} \mathbf{h}_{0}\right|_{\mathcal{S}}$ and from that

$$
\begin{aligned}
\widehat{\mathcal{S}} & \in \underset{\mathcal{S} \in \mathcal{C}_{k}}{\operatorname{argmin}}\left\|\left.\min \left\{1, r /\left\|\left.\mathbf{h}_{0}\right|_{\mathcal{S}}\right\|\right\} \mathbf{h}_{0}\right|_{\mathcal{S}}-\mathbf{h}_{0}\right\|^{2} \\
& =\underset{\mathcal{S} \in \mathcal{C}_{k}}{\operatorname{argmin}}\left\|\left.\min \left\{0, r /\left\|\left.\mathbf{h}_{0}\right|_{\mathcal{S}}\right\|-1\right\} \mathbf{h}_{0}\right|_{\mathcal{S}}\right\|^{2}+\left\|\left.\mathbf{h}_{0}\right|_{\mathcal{S}^{c}}\right\|^{2} \\
& =\underset{\mathcal{S} \in \mathcal{C}_{k}}{\operatorname{argmin}}\left(\left(1-r /\left\|\left.\mathbf{h}_{0}\right|_{\mathcal{S}}\right\|\right)_{+}^{2}-1\right)\left\|\left.\mathbf{h}_{0}\right|_{\mathcal{S}}\right\|^{2} \\
& =\underset{\mathcal{S} \in \mathcal{C}_{k}}{\operatorname{argmax}} q(\mathcal{S}):=\left\|\left.\mathbf{h}_{0}\right|_{\mathcal{S}}\right\|^{2}-\left(\left\|\left.\mathbf{h}_{0}\right|_{\mathcal{S}}\right\|-r\right)_{+}^{2} .
\end{aligned}
$$

Furthermore, let

$$
\mathcal{S}_{0}=\operatorname{supp}\left(\mathrm{P}_{\mathcal{C}_{\mathrm{k}},+\infty}\left[\mathbf{h}_{0}\right]\right)=\underset{\mathcal{S} \in \mathcal{C}_{k}}{\operatorname{argmax}}\left\|\left.\mathbf{h}_{0}\right|_{\mathcal{S}}\right\|
$$

If $\left\|\left.\mathbf{h}_{0}\right|_{\mathcal{S}_{0}}\right\| \leq r$ then $q(\mathcal{S})=\left\|\left.\mathbf{h}_{0}\right|_{\mathcal{S}}\right\| \leq q\left(\mathcal{S}_{0}\right)$ for any $\mathcal{S} \in \mathcal{C}_{k}$ and thereby $\widehat{\mathcal{S}}=\mathcal{S}_{0}$. Thus, we focus on cases that $\left\|\left.\mathbf{h}_{0}\right|_{\mathcal{S}_{0}}\right\|>r$ which implies $q\left(\mathcal{S}_{0}\right)=2\left\|\left.\mathbf{h}_{0}\right|_{\mathcal{S}_{0}}\right\| r-r^{2}$. For any $\mathcal{S} \in \mathcal{C}_{k}$ if $\left\|\left.\mathbf{h}_{0}\right|_{\mathcal{S}}\right\| \leq r$ we have $q(\mathcal{S})=\left\|\left.\mathbf{h}_{0}\right|_{\mathcal{S}}\right\|^{2} \leq r^{2}<2\left\|\left.\mathbf{h}_{0}\right|_{\mathcal{S}_{0}}\right\| r-r^{2}=$ $q\left(\mathcal{S}_{0}\right)$, and if $\left\|\left.\mathbf{h}_{0}\right|_{\mathcal{S}}\right\|>r$ we have $q(\mathcal{S})=2\left\|\left.\mathbf{h}_{0}\right|_{\mathcal{S}}\right\| r-r^{2} \leq 2\left\|\left.\mathbf{h}_{0}\right|_{\mathcal{S}_{0}}\right\| r-r^{2}=q\left(\mathcal{S}_{0}\right)$ where (8) is applied. Therefore, we have shown that $\widehat{\mathcal{S}}=\mathcal{S}_{0}$. It is then straightforward to show the desired result that projecting $\mathrm{P}_{\mathcal{C}_{k},+\infty}\left[\mathbf{h}_{0}\right]$ onto the centered sphere of radius $r$ yields $\mathrm{P}_{\mathcal{C}_{k}, r}\left[\mathbf{h}_{0}\right]$.

\section{REFERENCES}

[1] F. R. Bach, "Consistency of the group lasso and multiple kernel learning," Journal of Machine Learning Research, vol. 9, pp. 1179-1225, Jun. 2008.

[2] V. Roth and B. Fischer, "The Group-Lasso for generalized linear models: uniqueness of solutions and efficient algorithms," in Proceedings of the 25th International Conference on Machine learning, ser. ICML '08, New York, NY, USA, 2008, pp. 848-855.

[3] L. Jacob, G. Obozinski, and J. Vert, "Group lasso with overlap and graph lasso," in Proceedings of the 26th Annual International Conference on Machine Learning, ser. ICML '09, New York, NY, USA, 2009, pp. 433-440.

[4] R. G. Baraniuk, V. Cevher, M. F. Duarte, and C. Hegde, "Model-based compressive sensing," IEEE Transactions on Information Theory, vol. 56, no. 4, pp. 1982-2001, 2010.

[5] F. Bach, "Structured sparsity-inducing norms through submodular functions," in Advances in Neural Information Processing Systems 23, Vancouver, BC, Canada, Dec. 2010, pp. 118-126.

[6] R. Jenatton, J. Audibert, and F. Bach, "Structured variable selection with Sparsity-Inducing norms," Journal of Machine Learning Research, vol. 12, pp. 2777-2824, Oct. 2011.

[7] A. Kyrillidis and V. Cevher, "Combinatorial selection and least absolute shrinkage via the CLASH algorithm," in Information Theory Proceedings (ISIT), IEEE International Symposium on, Jul. 2012, pp. 2216-2220.

[8] V. Chandrasekaran, B. Recht, P. A. Parrilo, and A. S. Willsky, "The convex geometry of linear inverse problems," Foundation of Computational Mathematics, vol. 12, no. 6, pp. 805-849, 2012.

[9] F. Bach, R. Jenatton, J. Mairal, and G. Obozinski, "Structured sparsity through convex optimization," Statistical Science, vol. 27, no. 4, pp. 450-468, Nov. 2012.

[10] M. Duarte and Y. Eldar, "Structured compressed sensing: From theory to applications," Signal Processing, IEEE Transactions on, vol. 59, no. 9, pp. 4053-4085, Sep. 2011.

[11] A. Tewari, P. K. Ravikumar, and I. S. Dhillon, "Greedy algorithms for structurally constrained high dimensional problems," in Advances in Neural Information Processing Systems 24, J. Shawe-Taylor, R. Zemel, P. Bartlett, F. Pereira, and K. Weinberger, Eds., 2011, pp. $882-890$.

[12] T. Blumensath, "Compressed sensing with nonlinear observations," 2010, preprint. [Online]. Available: http://eprints.soton.ac.uk/164753/1/B_Nonlinear.pdf

[13] A. Lozano, G. Swirszcz, and N. Abe, "Group orthogonal matching pursuit for logistic regression," in the Fourteenth International Conference on Artificial Intelligence and Statistics, G. Gordon, D. Dunson, and M. Dudik, Eds., vol. 15. Ft. Lauderdale, FL, USA: JMLR W\&CP, 2011, pp. 452-460.

[14] Y. C. Pati, R. Rezaiifar, and P. S. Krishnaprasad, "Orthogonal matching pursuit: Recursive function approximation with applications to wavelet decomposition," in Conference Record of the 27th Asilomar Conf. on Signals, Systems and Computers, vol. 1, Pacific Grove, CA, Nov. 1993, pp. $40-44$.

[15] A. Beck and Y. C. Eldar, "Sparsity constrained nonlinear optimization: Optimality conditions and algorithms," SIAM Journal on Optimization, vol. 23, no. 3, pp. 1480-1509, 2013.

[16] T. Blumensath, "Compressed sensing with nonlinear observations and related nonlinear optimization problems," IEEE Transactions on Information Theory, vol. 59, no. 6, pp. 3466-3474, 2013.

[17] F. Bunea, "Honest variable selection in linear and logistic regression models via $\ell_{1}$ and $\ell_{1}+\ell_{2}$ penalization," Electronic Journal of Statistics, vol. 2 , pp. 1153-1194, 2008.

[18] S. Negahban, P. Ravikumar, M. Wainwright, and B. Yu, "A unified framework for high-dimensional analysis of $M$-estimators with decomposable regularizers," in Advances in Neural Information Processing Systems 22, Vancouver, BC, Canada, Dec. 2009, pp. 1348-1356, long version available at arXiv:1010.2731v1 [math.ST]

[19] A. Agarwal, S. Negahban, and M. Wainwright, "Fast global convergence rates of gradient methods for high-dimensional statistical recovery," in Advances in Neural Information Processing Systems 23, Vancouver, BC, Canada, 2010, pp. 37-45, long version available at arXiv:1104.4824v1 [stat.ML]

[20] S. M. Kakade, O. Shamir, K. Sridharan, and A. Tewari, "Learning exponential families in High-Dimensions: strong convexity and sparsity," in Proceedings of the 13th International Conference on Artificial Intelligence and Statistics, ser. JMLR Workshop and Conference Proceedings, vol. 9, Sardinia, Italy, 2010, pp. 381-388.

[21] C. Hegde, P. Indyk, and L. Schmidt, "Approximation-tolerant model-based compressive sensing," in Proceedings of the Twenty-Fifth Annual ACM-SIAM Symposium on Discrete Algorithms, 2014, ch. 113, pp. 1544-1561. 
[22] T. Blumensath, "Sampling and reconstructing signals from a union of linear subspaces," Information Theory, IEEE Transactions on, vol. 57, no. 7, pp. 4660-4671, 2011

[23] M. Davenport, D. Needell, and M. Wakin, "Signal space CoSaMP for sparse recovery with redundant dictionaries," IEEE Transactions on Information Theory, vol. 59, no. 10, pp. 6820-6829, 2013

[24] R. Giryes, S. Nam, M. Elad, R. Gribonval, and M. Davies, "Greedy-like algorithms for the cosparse analysis model," Linear Algebra and its Applications, Special Issue on Sparse Approximate Solution of Linear Systems, vol. 441, pp. 22-60, 2014.

[25] S. Bahmani, B. Raj, and P. Boufounos, "Greedy sparsity-constrained optimization," Journal of Machine Learning Research, vol. 14, no. 3, pp. 807-841, 2013.

[26] S. A. van de Geer, Empirical processes in M-estimation. Cambridge, UK: Cambridge University Press, 2000.

[27] V. Vapnik, Statistical learning theory. New York, NY: Wiley, 1998.

[28] A. J. Dobson and A. Barnett, An Introduction to Generalized Linear Models, 3rd ed. Boca Reaton, FL: Chapman and Hall/CRC, May 2008.

[29] J. A. Tropp, "User-friendly tail bounds for sums of random matrices," Foundations of Computational Mathematics, vol. 12, no. 4, pp. 389-434, 2012.

[30] D. Hsu, S. Kakade, and T. Zhang, "Tail inequalities for sums of random matrices that depend on the intrinsic dimension," Electron. Commun. Probab., vol. 17, no. 14, pp. 1-13, 2012. 